\title{
REPRESENTATIONS OF BANACH ALGEBRAS AS ALGEBRAS OF COMPLETELY BOUNDED MAPS
}

\author{
TIMUR OIKHBERG*
}

\begin{abstract}
We use the notion of $\Pi_{2}$-hyperreflexivity to construct, for a wide variety of Banach algebras $\mathscr{A}$, an operator space $X$ and a representation $\pi: \mathscr{A} \rightarrow C B(X)$, such that $C B(X)$ consists of 2 -summing perturbations of $\pi(\mathscr{A})$. This gives rise to some examples of operator spaces with interesting properties.
\end{abstract}

\section{Introduction}

In this paper, we try to answer two related questions.

(1) Suppose a unital Banach algebra $\mathscr{A}$, and a unital contractive faithful representation $\pi: \mathscr{A} \rightarrow B(E)$ ( $E$ is a Banach space), are given. Can we equip $E$ with an operator space structure $X$, such that $C B(X)=$ $\pi(\mathscr{A})+\Pi_{2}(X)$, where $\Pi_{2}(X)$ denotes the set of 2-summing operators on $X$ ?

(2) Suppose a unital Banach algebra $\mathscr{A}$ is given. Can we construct an operator space $X$, and a unital contractive faithful representation $\pi: \mathscr{A} \rightarrow$ $C B(X)$, such that $C B(X)=\pi(\mathscr{A})+\Pi_{2}(X)$ ?

Here, $\Pi_{2}$ is the ideal of 2-summing operators (see Section 2 for its definition and basic properties), and $C B(X)$ denotes the set of completely bounded maps on an operator space $X$ (see e.g. [13], [27], [31] for more information). For certain Banach algebras $\mathscr{A}$ (and certain representations $\pi$ ), we give a positive answer to the above questions in Section 3 (Theorem 3.1, Corollary 3.3). In Section 4, we construct further examples of Banach algebras $\mathscr{A}$, representations $\pi$, and operator spaces $X$ as above. This gives rise to operator spaces with unusual and interesting properties (Theorems $4.3,4.6$ ). The proofs require an investigation into hyperreflexivity of spaces and sets with respect to the ideal

\footnotetext{
* The author was partially supported by the NSF grant DMS-0500957.

Received February 23, 2008.
} 
$\Pi_{2}$. In Section 2, we review hyperreflexivity with respect to operator ideals, and prove some preliminary results.

In a slightly different context, the problems of representing Banach algebras in a "nice" way has recently been considered in [8], [9], [20], [36]. We briefly describe these results in Section 3 (Remark 3.7).

\section{Preliminary results on hyperreflexivity}

In this paper, we use the notion of hyperreflexivity with respect to an operator ideal, introduced in [23]. Suppose $\mathfrak{B}$ is a maximal Banach operator ideal. That is, for any pair $X, Y$ of Banach spaces, $\mathfrak{B}(X, Y)$ is a subset of $B(X, Y)$, equipped with the norm $\boldsymbol{\beta}(\cdot)$ in such a way that $(\mathcal{B}(X, Y), \boldsymbol{\beta})$ is a Banach space. By convention, we set $\beta(T)=\infty$ if $T \in B(X, Y) \backslash \mathfrak{B}(X, Y)$. The ideal property means that, for any Banach spaces $X_{0}, X, Y, Y_{0}$, and every $T \in \mathfrak{B}(X, Y), T_{X} \in B\left(X_{0}, X\right)$, and $T_{Y} \in B\left(Y, Y_{0}\right)$, we have $\beta\left(T_{Y} T T_{X}\right) \leqslant$ $\left\|T_{Y}\right\| \boldsymbol{\beta}(T)\left\|T_{X}\right\|$. The ideal $\mathfrak{B}$ is maximal if, for every $T \in B(X, Y), \boldsymbol{\beta}(T)=$ $\sup \boldsymbol{\beta}(q T i)$, where $i: E \rightarrow X$ is an injection, $q: Y \rightarrow F$ is a quotient, and the spaces $E$ and $F$ are finite dimensional. For further information about operator ideals, see [10], [11], [29], [35].

In this paper, we concentrate on the ideal $\Pi_{2}$ of 2-summing maps. Recall that the 2-summing norm of $T \in B(X, Y)$ (denoted by $\pi_{2}(T)$ ) is defined as the smallest positive constant $c$ such that, for every $x_{1}, \ldots, x_{n} \in X$,

$$
\left(\sum_{i}\left\|T x_{i}\right\|^{2}\right)^{1 / 2} \leqslant c \sup _{x^{*} \in X^{*},\left\|x^{*}\right\| \leqslant 1}\left(\sum_{i}\left|x^{*}\left(x_{i}\right)\right|^{2}\right)^{1 / 2} .
$$

The ideal of 2-summing operators is denoted by $\Pi_{2}$. If $X$ and $Y$ are Hilbert spaces, then it is well-known that $\Pi_{2}(X, Y)=\mathscr{S}_{2}(X, Y)$, and $\pi_{2}(T)=\|T\|_{2}$. Here, $\mathscr{T}_{2}$ and $\|\cdot\|_{2}$ denote the space of Hilbert-Schmidt maps, and the HilbertSchmidt norm, respectively.

Suppose $\mathscr{A}$ is a non-empty convex balanced subset of $B(X, Y)$, and $\mathfrak{B}$ is an operator ideal. For $T \in B(X, Y)$ denote by $d_{\mathscr{A}, \mathfrak{B}}(T)$ the infimum of all $\lambda>0$ with the property that, for any $\gamma \geqslant 1, \boldsymbol{\beta}(u T v) \leqslant \lambda \gamma$ whenever the contractions $v: E \rightarrow X$ and $u: Y \rightarrow F$ ( $E$ and $F$ are finite dimensional) satisfy $\boldsymbol{\beta}(u a v) \leqslant \gamma$ for every $a \in \mathscr{A}$. Equivalently, $d_{\mathscr{A}, \mathfrak{B}}(T)>\lambda$ iff there exists $\gamma \geqslant 1$ and contractions $u$, v s.t. $\boldsymbol{\beta}(u T v)>\lambda \gamma$, yet $\boldsymbol{\beta}($ uav $) \leqslant \gamma$ $\forall a \in \mathscr{A}$.

Observe that, for any $\lambda \in \mathcal{C}, a \in \mathscr{A}$, and $S \in \mathfrak{B}(E, F)$, we have $\beta(u(\lambda a+$ $S) v) \leqslant|\lambda| \gamma+\boldsymbol{\beta}(S)$ for any pair of contractions $u$, $v$, with the property that $\boldsymbol{\beta}($ uav $) \leqslant \gamma$ for every $a \in \mathscr{A}$. Thus,

$$
d_{\mathscr{A}, \mathfrak{B}}(\lambda a+S) \leqslant|\lambda|+\boldsymbol{\beta}(S) .
$$


The definition of Azoff-Shehada hyperreflexivity given below arose from an attempt to reverse this implication: namely, to conclude that any $T \in B(E, F)$ with finite $d_{\mathscr{A}, \mathfrak{B}}(T)$ belongs to $\mathscr{C} \mathscr{A}+\mathfrak{B}(E, F)$.

For $C_{1}, C_{2}>0$, we say that $\mathscr{A}$ is $\left(C_{1}, C_{2}\right)-\mathfrak{B}$-Azoff-Shehada hyperreflexive $\left(\left(C_{1}, C_{2}\right)-\mathfrak{B}\right.$-ASHR, for short) if, for any $T \in B(X, Y)$ with $d_{\mathscr{A}, \mathfrak{B}}(T)$ finite, and any $\varepsilon>0$, we can write $T=\lambda a+S$, with $a \in \mathscr{A}, \lambda \in \mathrm{C}$, $S \in \mathfrak{B}(X, Y),|\lambda|<C_{1} d_{\mathscr{A}, \mathfrak{B}}(T)+\varepsilon$, and and $\beta(S)<C_{2} d_{\mathscr{A}, \mathfrak{B}}(T)+\varepsilon . \mathscr{A}$ is said to be $\mathfrak{B}$-ASHR if it $\left(C_{1}, C_{2}\right)-\mathfrak{B}$-ASHR for some $C_{1}$ and $C_{2}$.

Throughout the paper, we work with maximal Banach ideals. In this case, the condition that $E$ and $F$ are finite dimensional is redundant.

Now suppose $\mathscr{A} \hookrightarrow B(X, Y)$ (throughout the paper, we use the notation " $Z_{1} \hookrightarrow Z_{2}$ " to mean " $Z_{1}$ is a closed linear subspace of a Banach space $Z_{2}$ "). It is easy to see that, if $\mathscr{A}$ is $\left(C^{\prime}, C\right)-\mathfrak{B}$-ASHR, then it is $(c, C)-\mathfrak{B}$-ASHR for any $c>0$ (indeed, in the definition above, $|\lambda|$ can be made arbitrarily small). In this case, we say that $\mathscr{A}$ is $C-\mathfrak{B}$-hyperreflexive. $\mathscr{A}$ is called $\mathfrak{B}$-hyperreflexive if it is $C-\mathfrak{B}$-hyperreflexive for some $C$. It as observed in [23] that $\mathscr{A}$ is $C-\mathfrak{B}$ hyperreflexive iff inf $\operatorname{a\in \mathscr {A}}_{\boldsymbol{\beta}} \boldsymbol{\beta}(T-a) \leqslant C d_{\mathscr{A}, \mathfrak{B}}(T)$, and $d_{\mathscr{A}, \mathfrak{B}}(T)=\sup \boldsymbol{\beta}(u T v)$, with the sup taken over all finite rank contractions $u$ and $v$ with $u \mathscr{A} v=0$. Note that, in this case, $d_{\mathscr{A}, \mathfrak{B}}(T)=\sup \boldsymbol{\beta}(q T i)$, where the supremum runs over all injections $i: E \rightarrow X$ and quotient maps $q: Y \rightarrow F$, for which $E$ and $F$ are finite dimensional, and $q \mathscr{A} i=0$.

The definitions presented above were introduced in [23] (although the definition of the Azoff-Shehada hyperreflexivity given there is slightly different). Numerous examples of sets which are, and are not, ASHR, can be found there. The same paper explains the connection with the classical notion of hyperreflexivity (see e.g. [6], [7], [19] for more information).

The following proposition (essentially proved in [23]) illustrates the connection between the connection between the hyperreflexivity of a space, and the ASHR of its unit ball.

Proposition 2.1. Suppose $(\mathfrak{B}, \boldsymbol{\beta})$ is a maximal ideal, and let $\mathscr{A}$ be a $C-\mathfrak{B}$-hyperreflexive subspace of $B(X, Y)$. Then $\mathrm{Ba}(\mathscr{A})$ is $(C+1, C)-\mathfrak{B}$ Azoff-Shehada hyperreflexive.

As in the classical theory of hyperreflexivity, we can use ampliations to create hyperreflexive spaces (or ASHR sets). To this end, consider a symmetric sequence space $\mathscr{E}$ (that is, a space with a 1 -symmetric basis). Denote by $\mathscr{E}_{n}$ $(n \in \mathrm{N})$ the span of $n$ elements of the canonical basis of $\mathscr{E}$. For $T \in B(E, F)$ and $n \in \mathrm{N} \cup\{\infty\}$, the $n$-th ampliation of $T($ on $\mathscr{E})$ is defined as $T^{(n, \mathscr{E})}=$ $I_{\mathscr{E}} \otimes T \in B(\mathscr{E}(E), \mathscr{E}(F))$. For $\mathscr{A} \subset B(E, F)$, set $\mathscr{A}^{(n, \mathscr{E})}=\left\{T^{(n, \mathscr{E})} \mid T \in \mathscr{A}\right\}$. When there is no possibility of confusion, we will omit $\mathscr{E}$, and simply write $T^{(n)}$ and $\mathscr{A}^{(n)}$. 
Theorem 2.2. Suppose $\mathscr{A}$ is a convex balanced subset subset of the unit ball of $B(E, F)$ ( $E$ and $F$ are Banach spaces), closed in the topology induced by $E \widehat{\otimes} F^{*}$. Suppose, furthermore, that $(\mathfrak{B}, \boldsymbol{\beta})$ is a maximal Banach operator ideal, and $\lim _{n} \boldsymbol{\beta}\left(I_{\mathscr{E}_{n}}\right)=\infty$, where $\mathscr{E}$ is $\ell_{p}(1 \leqslant p<\infty)$ or $c_{0}$. Then $\mathscr{A}^{(\infty, \mathscr{E})}$ is a $(5,25)-\mathfrak{B}$-ASHR subset of $B(\mathscr{E}(E), \mathscr{E}(F))$.

The lemmas below lead to the proof of Theorem 2.2. For the sake of brevity, introduce some notation. For $\mathscr{E}$ as above, $\tilde{E}=\mathscr{E}(E)$ and $\tilde{F}=\mathscr{E}(F)$. For $\mathscr{F} \subset \mathrm{N}$, define the contractive projection $P_{\mathscr{F}} \in B(\mathscr{E})$ by setting

$$
P_{\mathscr{F}} \delta_{i}= \begin{cases}\delta_{i} & i \in \mathscr{F} \\ 0 & i \notin \mathscr{F}\end{cases}
$$

where $\left(\delta_{i}\right)$ is the canonical basis for $\mathscr{E}$. Abusing the notation somewhat, we use $P_{\mathscr{F}}$ for $P_{\mathscr{F}} \otimes I_{E} \in B(\tilde{E})$ and $P_{\mathscr{F}} \otimes I_{F} \in B(\tilde{F})$. We denote by $Q_{n}$ the projection $P_{\{1, \ldots, n\}}$ on the first $n$ elements of the basis of $\mathscr{E}$ (with the conventions described above). An operator $T \in B(\tilde{E}, \tilde{F})$ is called diagonal if $T=\sum_{i} P_{\{i\}} T P_{\{i\}}$ (convergence in the strong operator topology). We also write $T=\left(\operatorname{diag}\left(P_{\{i\}} T P_{\{i\}}\right)\right)$.

Lemma 2.3. Suppose $\mathscr{E}$ is an unconditional sequence space, $\mathfrak{B}$ is a maximal operator ideal, $E$ and $F$ are Banach spaces, and $\mathscr{A} \subset B(E, F)$ is closed in the topology induced by $E \widehat{\otimes} F^{*}$. Suppose, furthermore, that $T^{\prime} \in B(\tilde{E}, \tilde{F})$ satisfies $d_{\mathscr{A}(\infty), \mathfrak{B}}\left(T^{\prime}\right)<1$. Then there exists a diagonal operator $T$ s.t. $\boldsymbol{\beta}\left(T-T^{\prime}\right) \leqslant 4$. Consequently, $d_{\mathscr{A}(\infty), \mathfrak{B}}(T)<5$.

Proof. Denote by $T$ the diagonal part of $T^{\prime}$, that is $T=\sum_{n} P_{\{n\}} T^{\prime} P_{\{n\}}$ (convergence in the strong operator topology). Let $T^{\prime \prime}=T^{\prime}-T$. For any $n \in \mathrm{N}$,

$$
Q_{n} T^{\prime \prime} Q_{n}=2^{2-n} \sum_{\mathscr{F} \subset\{1, \ldots, n\}}\left(Q_{n}-P_{\mathscr{F}}\right) T^{\prime} P_{\mathscr{F}} .
$$

However, $\left(Q_{n}-P_{\mathscr{F}}\right) B(E, F)^{(\infty)} P_{\mathscr{F}}=0$, hence $\boldsymbol{\beta}\left(\left(Q_{n}-P_{\mathscr{F}}\right) T^{\prime} P_{\mathscr{F}}\right)<1$. Therefore, $\boldsymbol{\beta}\left(Q_{n} T^{\prime \prime} Q_{n}\right)<4$ for every $n$.

By the maximality of $\mathfrak{B}, \boldsymbol{\beta}\left(T^{\prime \prime}\right) \leqslant 4$. Indeed, otherwise there exists a finite rank contraction $u$ s.t. $\boldsymbol{\beta}\left(T^{\prime \prime} u\right)>4$. However, $\lim _{n}\left(Q_{n} u-u\right)=0$ (this follows from the fact that, for any finite rank map $S, \boldsymbol{\beta}(S) \leqslant v_{1}(S)$, where $v_{1}(\cdot)$ denotes the nuclear norm). Therefore, we can assume that $Q_{m} u=u$ ( $m$ is sufficiently large). In a similar fashion, we find $n \geqslant m$ s.t. $\beta\left(Q_{n} T^{\prime \prime} Q_{m} u\right)>4$. Therefore, $\boldsymbol{\beta}\left(Q_{n} T^{\prime \prime} Q_{n}\right)>4$, which contradicts the results of the previous paragraph.

By $(2.1), d_{\mathscr{A}(\infty), \mathfrak{B}}(T)<5$.

Lemma 2.4. Suppose $\mathscr{E}$ is a symmetric sequence space, $\mathfrak{B}, E, F$, and $\mathscr{A}$ are as in the previous lemma. Suppose, furthermore, $T=\operatorname{diag}\left(T_{i}\right) \in B(\tilde{E}, \tilde{F})$ is a 
diagonal operator, and $d_{\mathscr{A}(\infty), \mathfrak{B}}(T)$ is finite. Then the sequence $\left(T_{i}\right)$ converges in norm.

Proof. Without loss of generality, $d_{\mathscr{A}(\infty), \mathfrak{B}}(T)<1$. We show that there exists $T_{0} \in B(E, F)$ s.t. $\lim _{i}\left\|T_{i}-T_{0}\right\|=0$. Indeed, suppose otherwise. Then there exist $c>0$ a sequence $n_{1}<m_{1}<n_{2}<m_{2}<\cdots$, and a sequence of norm-1 vectors $e_{i} \in E$, s.t. $\left\|\left(T_{n_{i}}-T_{m_{i}}\right) e_{i}\right\|>c$ for every $i$. Consequently, we can find a sequence of norm- 1 vectors $f_{i}^{*} \in F^{*}$, s.t. $\left\langle\left(T_{n_{i}}-T_{m_{i}}\right) e_{i}, f_{i}^{*}\right\rangle>c$.

Fix $N \in \mathrm{N}$ s.t. $\boldsymbol{\beta}\left(I_{\mathscr{E}_{N}}\right)>4 / c$. Denote by $\left(\delta_{i}\right)_{i=1}^{\infty}$ the canonical basis of $\mathscr{E}$. Consider the contractions $u: \mathscr{E}_{N}=\operatorname{span}\left[\delta_{1}, \ldots, \delta_{N}\right] \rightarrow \tilde{E}$ and $v: \tilde{F} \rightarrow \mathscr{E}_{N}$, defined by

$$
u \delta_{i}=e_{i} \otimes\left(\delta_{n_{i}}+\delta_{m_{i}}\right) / 2, \quad v\left(\sum_{j} f_{j} \otimes \delta_{j}\right)=\sum_{i=1}^{N} \frac{1}{2}\left\langle f_{n_{i}}-f_{m_{i}}, f_{i}^{*}\right\rangle \delta_{i} .
$$

Then $v \mathscr{A}^{(\infty)} u=0$, and $v T u \delta_{i}=c_{i} \delta_{i}$, where, for each $i, c_{i} \geqslant c / 4$. Therefore,

$$
1>\boldsymbol{\beta}(v T u) \geqslant \frac{c}{4} \boldsymbol{\beta}\left(I_{\mathscr{E}_{N}}\right)>1,
$$

a contradiction.

Consider $T=\operatorname{diag}\left(T_{i}\right) \in B(\tilde{E}, \tilde{F})$, for which $d_{\mathscr{A}(\infty), \mathfrak{B}}(T)$ is finite. By the previous result, $T_{0}=\lim T_{i}$ exists. For brevity, write $\tilde{T}=T_{0}^{(\infty)}$.

Lemma 2.5. Suppose $E, F, \mathscr{A}, \mathscr{E}$, and $\mathfrak{B}$ are as above, and the diagonal operator $T \in B(\tilde{E}, \tilde{F})$ satisfies $d_{\mathscr{A}(\infty), \mathfrak{B}}(T)<1$. Then $\boldsymbol{\beta}(T-\tilde{T}) \leqslant 4$, and $d_{\mathscr{A}(\infty), \mathfrak{B}}(\tilde{T})<1$.

Proof. Suppose, for the sake of contradiction, $\boldsymbol{\beta}(T-\tilde{T})>4$. Then $\boldsymbol{\beta}\left(Q_{n}(T-\tilde{T}) Q_{n}\right)>4$ for some $n$. Find a finite rank contraction $u_{0}: E_{0} \rightarrow$ $\operatorname{ran} Q_{n}$ s.t. $\beta\left(Q_{n}(T-\tilde{T}) Q_{n} u_{0}\right)>4$. Fix $\varepsilon>0$, and find $N>n$ so large that $\left\|T_{i}-T_{0}\right\|<\varepsilon /\left(n\right.$ rank $\left.u_{0}\right)$ for $i>N$. Let $S_{E}$ be the right shift operator on $\tilde{E}$, defined by $S_{E}\left(\left(e_{1}, e_{2}, \ldots\right)\right)=\left(0, e_{1}, e_{2}, \ldots\right)$. Then $\boldsymbol{\beta}\left((T-\tilde{T}) S_{E}^{N} Q_{n} u_{0}\right)<\varepsilon$.

Let $u=\left(u_{0}+S_{E}^{N} u_{0}\right) / 2$. Define $v \in B(\tilde{F})$ by setting

$$
v\left(\left(f_{1}, f_{2}, \ldots\right)\right)=\frac{1}{2}\left(f_{1}-f_{N+1}, \ldots, f_{n}-f_{N+n}, 0,0, \ldots\right)
$$

Then $u$ and $v$ are contractions, satisfying $v B(E, F)^{(\infty)} u=0$ Furthermore,

$$
v T u=\frac{1}{2} v(T-\tilde{T})\left(u_{0}+S_{E}^{N} u_{0}\right)=\frac{1}{4}\left((T-\tilde{T}) u_{0}+\left(S_{F}^{N}\right)^{-1}(T-\tilde{T}) S_{E}^{N} u_{0}\right),
$$


where $S_{F}$ is defined similarly to $S_{E}$. However, $\boldsymbol{\beta}\left((T-\tilde{T}) S_{E}^{N} u_{0}\right)<\varepsilon$, hence $\boldsymbol{\beta}(v T u)>1-\varepsilon / 4$. As $\varepsilon$ is an arbitrary positive number, we arrive at a contradiction.

Now suppose $u$ and $v$ are finite rank contractions s.t. $\beta\left(v a^{(\infty)} u\right) \leqslant \gamma$ $(\gamma \geqslant 1)$ for any $a \in \mathscr{A}$. We shall show that $\boldsymbol{\beta}(v \tilde{T} u) \leqslant \gamma$. Let $v_{N}=S_{F}^{-N}$ and $u_{N}=S_{E}^{N}$. Then, for any $N, \boldsymbol{\beta}\left(v b^{(\infty)} u\right)=\boldsymbol{\beta}\left(v_{N} b^{(\infty)} u_{N}\right)$ for any $b \in B(E, F)$, hence, in particular, $\boldsymbol{\beta}\left(v_{N} a^{(\infty)} u_{N}\right) \leqslant \gamma$. On the other hand, $\lim _{N} \boldsymbol{\beta}((T-$ $\left.\tilde{T}) u_{N}\right)=0$, and therefore,

$$
\boldsymbol{\beta}(v \tilde{T} u)=\boldsymbol{\beta}\left(v_{N} \tilde{T} u_{N}\right)=\lim _{N} \boldsymbol{\beta}\left(v_{N} T u_{N}\right) \leqslant \gamma,
$$

as desired.

Lemma 2.6. Suppose, $E, F, \mathscr{E}, \mathfrak{B}$, and $\mathscr{A}$ as above, and $T \in B(E, F)$ is such that $d_{\mathscr{A}(\infty), \mathfrak{B}}\left(T^{(\infty)}\right)<1$. Then $T \in \mathscr{A}$.

Proof. As $\mathscr{A}$ is convex, balanced, and ( $\left.B(E, F), E \widehat{\otimes} F^{*}\right)$-closed, there exist $c>0$ and a norm- $1 s \in E \widehat{\otimes} F^{*}$, s.t. $|\langle a, s\rangle|<c$ for any $a \in \mathscr{A}$, yet $\langle T, s\rangle>$ $c$. Moreover, we can always assume that $s$ belongs to the algebraic tensor product $E \otimes F^{*}$. Write $s=\sum_{i=1}^{N} \gamma_{i} e_{i} \otimes f_{i}^{*}$, where $e_{1}, \ldots, e_{N}, f_{1}^{*}, \ldots, f_{N}^{*}$ are are norm- 1 elements of $E$ and $F^{*}$, respectively, the numbers $\left(\gamma_{i}\right)$ are positive, adding up to 1 . We can write $\gamma_{i}=\alpha_{i} \beta_{i}$, where the positive scalars $\left(\alpha_{i}\right)$ and $\left(\beta_{i}\right)$ satisfy $\left\|\left(\alpha_{i}\right)_{i=1}^{N}\right\|_{\mathscr{E}}=1=\left\|\left(\beta_{i}\right)_{i=1}^{N}\right\|_{\mathscr{E} *}$.

Fix $M>2 / c$, and define the linear maps $u: \mathscr{E}_{M} \rightarrow \tilde{E}$ and $v: \tilde{F} \rightarrow \mathscr{E}_{M}$ by

$$
u \delta_{i}=\sum_{j=1}^{N} \alpha_{j} \delta_{N i+j} \otimes e_{i}, \quad v\left(\sum_{j} \delta_{j} \otimes f_{j}\right)=\sum_{i=1}^{M} \sum_{j=1}^{N} \beta_{j}\left\langle f_{N i+j}, f_{j}^{*}\right\rangle \delta_{i}
$$

(here, as before, $\left(\delta_{i}\right)$ stands for the canonical basis of $\mathscr{E}$ ). Clearly, $u$ and $v$ are contractions. For any $a \in B(E, F), v a^{(\infty)} u=\operatorname{diag}(\langle a, s\rangle$ ) (repeated $M$ times). Thus, for $a \in \mathscr{A}, \boldsymbol{\beta}\left(v a^{(\infty)} u\right)<c M \boldsymbol{\beta}\left(I_{\mathscr{E}_{N}}\right)$, and $\boldsymbol{\beta}\left(v T^{(\infty)} u\right)>$ $c M \boldsymbol{\beta}\left(I_{\mathscr{E}_{N}}\right)$. This contradicts our assumption that $d_{\mathscr{A}(\infty), \mathfrak{B}}\left(T^{(\infty)}\right)<1$.

Proof of Theorem 2.2. Suppose $T^{\prime} \in B(\mathscr{E}(E), \mathscr{E}(F))$ is such that $d_{\mathscr{A}, \mathfrak{B}}\left(T^{\prime}\right)<1 / 5$. By Lemma 2.3 , there exists a diagonal $T \in B(\mathscr{E}(E), \mathscr{E}(F))$ s.t. $\boldsymbol{\beta}\left(T-T^{\prime}\right)<4 / 5$, and $d_{\mathscr{A}, \mathfrak{B}}(T)<1$. By Lemmas 2.5 and 2.6, there exists $a \in \mathscr{A}$ s.t. $\boldsymbol{\beta}\left(T-a^{(\infty)}\right) \leqslant 4$. Therefore, $\boldsymbol{\beta}\left(T^{\prime}-a^{(\infty)}\right) \leqslant 24 / 5$.

\section{Representations of Banach algebras}

In this section, we apply $\Pi_{2}$-ASHR to constructing operator spaces with prescribed families of c.b. maps (see e.g. [21], [22], [24], [25] for other work in this direction). More precisely, for a given subset $\mathscr{A}$ of $B(E)$, we seek to 
equip $E$ with an operator space structure $X$ s.t. $C B(X)$ consists of 2-summing perturbations of $C \mathscr{A}$. Furthermore, for a given unital Banach algebra $\mathscr{A}$, we look for a representation $\pi: \mathscr{A} \rightarrow C B(X)$, s.t. $C B(X)=\pi(\mathscr{A})+\Pi_{2}(X)$. 2summing operators are strictly singular, hence $C B(X)$ is comprised of "small perturbations" of $\pi(\mathscr{A})$.

Theorem 3.1. Suppose $E$ is a Banach space with separable dual, and $\mathscr{A}$ is a balanced convex subset of $\mathrm{Ba}(B(E))$, closed in the topology induced by $E \widehat{\otimes} E^{*}$, containing the identity, and such that $a b \in \mathscr{A}$ whenever $a, b \in \mathscr{A}$. Suppose, furthermore, that $\mathscr{A}$ is $\left(C_{1}, C_{2}\right)-\Pi_{2}$-ASHR. Then there exists an operator space $X$, isometric to $E$, such that $C B(X)=C \mathscr{A}+\Pi_{2}(E)$. More precisely:

(1) If $a \in \mathscr{A}$, then $\|a\|_{c b} \leqslant 1$. If $S \in \Pi_{2}(X)$, then $\|S\|_{c b} \leqslant \pi_{2}(S)$.

(2) If $T \in C B(X)$, then there exists $S \in \Pi_{2}(X)$ and $a \in \mathscr{A}$, so that $T=\lambda a+S, a \in \mathscr{A},|\lambda| \leqslant 4 C_{1}\|T\|_{c b}$, and $\pi_{2}(S) \leqslant 4 C_{2}\|T\|_{c b}$.

Remark 3.2. Proposition 2.1 shows that the above theorem can be applied when $\mathscr{A}$ is a $\Pi_{2}$-hyperreflexive subspace of $B(E)$.

An application of Theorem 2.2 immediately yields:

Corollary 3.3. Suppose $E$ is a Banach space with separable dual, and $\mathscr{A}$ is a convex balanced subset of $\mathrm{Ba}(B(E))$, closed in the topology induced by $E \widehat{\otimes} E^{*}$, containing the identity, and such that $a b \in \mathscr{A}$ whenever $a, b \in \mathscr{A}$. Denote by $\mathscr{E}$ the sequence space $\ell_{p}(1<p<\infty)$ or $c_{0}$. Then there exists an operator space $X$, isometric to $\mathscr{E}(E)$, such that $C B(X)=\mathscr{A}^{(\infty)}+\Pi_{2}(X)$. More precisely:

(1) If $a \in \mathscr{A}$, then $\left\|a^{(\infty)}\right\|_{c b} \leqslant 1$. If $S \in \Pi_{2}(X)$, then $\|S\|_{c b} \leqslant \pi_{2}(S)$.

(2) If $T \in C B(X)$, then there exists $S \in \Pi_{2}(X)$ and $a \in \mathscr{A}$, so that $T=\lambda a^{(\infty)}+S, a \in \mathscr{A},|\lambda| \leqslant 25\|T\|_{c b}$, and $\pi_{2}(S) \leqslant 120\|T\|_{c b}$.

Note that the decomposition $T=a^{(\infty)}+S$ is unique, since any 2-summing operator is strictly singular.

In our previous work (see [23] and references therein), we dealt with representations of Banach algebras on Hilbertian operator spaces. However, for a given algebra, non-trivial representations on a Hilbert space need not exist (see [3]). Thus, the results of this paper cover a wider class of Banach algebras.

Recall that a dual Banach algebra is a Banach algebra which is dual as a Banach space, such that multiplication is separately weak* continuous.

Corollary 3.4. Suppose $\mathscr{A}$ is a dual Banach algebra with a separable predual. Then there exists a separable reflexive Banach space $E$, a unital isometric weak ${ }^{*}$-to-weak ${ }^{*}$ continuous representation $\pi: \mathscr{A} \rightarrow B(E)$, and an 
operator space $X$, isometric to $\ell_{2}(E)$, such that $C B(X)=\pi(\mathscr{A}) \otimes I_{\ell_{2}}+\Pi_{2}(X)$. More precisely, $T \in B(X)$ is completely bounded if and only if $T=\pi(a) \otimes$ $I_{\ell_{2}}+S$, with $a \in \mathscr{A}$ and $S \in \Pi_{2}(X)$.

(1) If $S \in \Pi_{2}(X)$, then $\|S\|_{c b} \leqslant \pi_{2}(S)$. If $a \in \mathscr{A}$, then $\|\pi(a)\|_{c b}=\|a\|$.

(2) Every $T \in C B(X)$ can be written as $T=\pi(a)+b$, with $a \in \mathscr{A}$, $S \in \Pi_{2}(X), \pi_{2}(S) \leqslant 120\|T\|_{c b}$, and $\|a\| \leqslant\|T\|$.

Remark 3.5. Reasoning as in [25], we can show that the space $X$ we construct in the proof of this corollary fails the Operator Approximation Property (OAP). Examples of other "interesting" spacing without the OAP can be found, for instance, in [23], [24].

Remark 3.6. The term $\Pi_{2}(X)$ in the statement of Corollary 3.4 cannot be avoided. Indeed, $\ell_{1}(Z)$, equipped with its convolution product, is a dual Banach algebra (see Section 4.4 of [33] for further results in this direction). However, the space $C B(X)$ cannot be isomorphic (in the Banach algebra sense) to $\ell_{1}(\mathrm{Z})$. Indeed, if this were the case, $X$ would need to be infinite dimensional. As any finite dimensional subspace of $X$ is completely complemented, $C B(X)$ contains infinitely many idempotents. However, $\ell_{1}(Z)$ contains only two idempotents: 0 and 1.

Remark 3.7. The Gelfand-Naimark-Segal theorem shows that every $C^{*}$ algebra can be represented as an algebra of operators on a Hilbert space. For a general Banach algebra, however, non-trivial representations on a Hilbert space need not exist [3]. Thus, we have to consider representations on larger classes of spaces. Recently, several results reminiscent of Corollary 3.4 appeared. M. Daws [8] showed that any dual Banach algebra has an isometric representation on $E$, for some Banach space $E$. M. Daws [9] and, independently, F. Uygul [36] showed that every completely contractive dual Banach algebra is completely isometric to a weak ${ }^{*}$-closed subalgebra of $C B(X)$, for some reflexive operator space $X$. However, in these constructions, a generic element of $B(E)$ or $C B(X)$ need not be a "small perturbation" of the image of an element of the algebra.

Finally, [20] gives examples of "natural" representations of certain algebras $\mathscr{A}$, arising from a non-abelian group $G$, of the form $\pi: \mathscr{A} \rightarrow C B\left(B\left(L_{2}(G)\right)\right)$. Here, once again, the small perturbations of $\pi(\mathscr{A})$ do not "fill" $C B\left(B\left(L_{2}(G)\right)\right)$.

The proof of Corollary 3.4 follows from the fact that any 2-summing operator is strictly singular, and the following lemma:

Lemma 3.8. Suppose $\mathscr{A}$ is a dual unital Banach algebra with a separable predual. Then there exists a separable reflexive Banach space $E$, and a unital contractive weak ${ }^{*}$-to-weak ${ }^{*}$ continuous representation $\pi: \mathscr{A} \rightarrow B(E)$. 
Proof. A recent result of M. Daws [8] states that, for any dual Banach algebra, $\mathscr{A}$, there exists a weak ${ }^{*}$-to-weak* continuous isometric representation $\psi: \mathscr{A} \rightarrow B(F)$, where $F$ is a reflexive Banach space. We first show that $F$ may be selected to be separable if $\mathscr{A}$ is separable in the weak* topology.

The existence of a countable fundamental, total, and bounded biorthogonal system in $\left(\mathscr{A}_{*}, \mathscr{A}\right)$ (see [26], [28]) implies the existence of a weak*-dense sequence $\left(a_{n}\right)_{n \in \mathrm{N}}$ in $\psi(\mathscr{A})$. For each $n, m \in \mathrm{N}$, find a norm one $f_{n, m} \in F$ s.t. $\left\|a_{n} f_{n, m}\right\| \geqslant\left\|a_{n}\right\| /\left(1+2^{-m}\right)$. Let $F_{1}=\operatorname{span} f_{1,1}$, and, for $n>1$,

$$
F_{n}=\operatorname{span}\left[a_{k} f_{i, j}, a_{k} F_{n-1}, F_{n-1} \mid 1 \leqslant i, j, k, \leqslant n\right] .
$$

Clearly, $F_{n}$ is finite dimensional, hence $G=\overline{\bigcup_{n} F_{n}}$ is separable. Moreover, $a_{k} F_{n} \subset F_{n+1}$ for $1 \leqslant k \leqslant n$, hence $a_{k} G \subset G$ for each $k$. We shall show that $a G \subset G$ for every $a \in \psi(\mathscr{A})$. Indeed, suppose $a f \notin G$ for some $f \in G$. Pick $f^{*} \in F^{*}$ s.t. $\left.f^{*}\right|_{G}=0$, and $\left\langle f^{*}, a f\right\rangle \neq 0$. Pick a sequence $\left(n_{k}\right)_{k \in \mathrm{N}}$ s.t. $a_{n_{k}} \rightarrow a$ in the weak* topology. In particular,

$$
0=\left\langle f^{*}, a_{n_{k}} f\right\rangle \rightarrow\left\langle f^{*}, a f\right\rangle \neq 0
$$

a contradiction.

Define $\phi: \mathscr{A} \rightarrow B(G)$ by setting, for $a \in \mathscr{A}, \phi(a)=\left.\psi(a)\right|_{G}$. Clearly, $\phi$ is an isometric representation. To show that $\phi$ is weak ${ }^{*}$-to-weak ${ }^{*}$ continuous, suppose $a_{i} \rightarrow 0$ in the weak* topology of $\mathscr{A}$, and show that $\lim _{i} \sum_{k=1}^{\infty}\left\langle g_{k}^{*}, \phi(a) g_{k}\right\rangle$ $=0$ whenever $\left(g_{k}\right) \subset G$ and $\left(g_{k}^{*}\right) \subset G^{*}$ satisfy $\sum_{k}\left\|g_{k}\right\|\left\|g_{k}^{*}\right\|<\infty$. Find $\left(f_{k}^{*}\right) \subset F^{*}$ s.t. $\left\|f_{k}^{*}\right\|=\left\|g_{k}^{*}\right\|$, and $\left.f_{k}^{*}\right|_{G}=g_{k}^{*}$. Recall that $\psi\left(a_{i}\right) \rightarrow \psi(a)$ weak* in $B(F)$, hence

$$
\sum_{k=1}^{\infty}\left\langle g_{k}^{*}, \phi(a) g_{k}\right\rangle=\sum_{k=1}^{\infty}\left\langle f_{k}^{*}, \psi(a) g_{k}\right\rangle \rightarrow 0 .
$$

Now denote by $e$ the identity in $\mathscr{A}$. Then $p=\phi(e)$ is a norm 1 idempotent in $B(G)$. Let $E=p(G)$, and define $\pi: \mathscr{A} \rightarrow B(E)$ by setting $\pi(a)=\left.\phi(a)\right|_{E}$. It is easy to show that $\pi$ is isometric, unital, and weak*-to-weak* continuous.

The proof of Theorem 3.1 uses some ideas of [23]. To define the space $X$, pick a sequence $\left(n_{i}\right)_{i=1}^{\infty} \subset \mathrm{N}$, in which every positive integer occurs infinitely many times. By [25], there exists a family $\left(E_{i}\right)_{i=1}^{\infty}$ of finite dimensional operator spaces such that: (i) $E_{i}$ is isometric to $\ell_{2}^{n_{i}}$, and (ii) for any operator $u: E_{i}^{*} \rightarrow E_{j}$, we have $\|u\|_{1} /\left(4+2^{-i}\right) \leqslant\|u\|_{c b} \leqslant\|u\|_{1}$ if $i=j$, $\|u\|_{c b}=\|u\|_{2}$ if $i \neq j$. Here, $\|\cdot\|_{1}$ and $\|\cdot\|_{2}$ are, respectively, the trace class norm and the Hilbert-Schmidt norm on operators between Hilbert spaces (they coincide with $v_{1}(\cdot)$ and $\pi_{2}(\cdot)$ ). 
Find a sequence of operators $u_{i}: E \rightarrow \ell_{2}^{n_{i}}$ such that $\pi_{2}\left(u_{i}\right)=1$ and, for any $\varepsilon>0, n \in \mathrm{N}$, and $u: E \rightarrow \ell_{2}^{n}$ with $\pi_{2}(u)=1$, there exists $i \in \mathrm{N}$ for which $n_{i}=n$ and $v_{1}\left(u_{i}-u\right)<\varepsilon$. We identify the range of $u_{i}$ with the space $E_{i}$ described above. Denote by $\mathscr{K}_{0}$ the space of compact operators on $\ell_{2}$ with finitely many non-zero entries. We define the operator space $X$ as follows: for $x \in E \otimes \mathscr{K}_{0}$, let

$$
\|x\|_{X \otimes \mathscr{K}_{0}}=\sup \left\{\left\|\left(u_{i} a \otimes I_{\mathscr{K}_{0}}\right) x\right\|_{E_{i} \otimes \mathscr{K}_{0}} \mid i \in \mathrm{N}, a \in \mathscr{A}\right\} .
$$

In other words, $X$ is Banach isometric to the space $E$, embedded into $\left(\sum_{i} E_{i}\right)_{\infty}$ via the map $e \rightarrow\left(u_{i} e\right)_{i}$. Therefore, $X$ is an operator space (Ruan's axioms hold). It is easy to see that $X$ is isometric to $E$ (as a Banach space). $\mathscr{A}$ is a unital semigroup, hence $\|a\|_{c b} \leqslant 1$ for any $a \in \mathscr{A}$. Moreover, all 2-summing operators on $X$ are completely bounded:

LeMmA 3.9. If $Y$ is an operator space, and $T: Y \rightarrow X$ is a 2-summing operator, then $\|T\|_{c b} \leqslant \pi_{2}(T)$.

Proof. By (3.1),

$$
\|T\|_{c b}=\sup \left\{\left\|u_{i} a T\right\|_{c b} \mid i \in \mathrm{N}, a \in \mathscr{A}\right\} \leqslant \sup \left\{v_{1}\left(u_{i} a T\right) \mid i \in \mathrm{N}, a \in \mathscr{A}\right\} .
$$

We have to show that, for any Banach spaces $X$ and $Y, u \in B(Y, X)$, and $v \in B\left(X, \ell_{2}^{n}\right)$, we have $v_{1}(v u) \leqslant \pi_{2}(v) \pi_{2}(u)$. By duality,

$$
v_{1}(v u)=\sup \left\{\operatorname{tr}(v u w) \mid w \in B\left(\ell_{2}^{n}, Y\right),\|w\| \leqslant 1\right\} .
$$

By Lemma 1.16 of [30], $\operatorname{tr}(v u w) \leqslant \pi_{2}(v) \pi_{2}(u w) \leqslant \pi_{2}(v) \pi_{2}(u)$.

To estimate the c.b. norms of operators from below, we need:

Lemma 3.10. Suppose $Y$ is a subspace of $X$. Consider the operators $T$ : $Y \rightarrow X, u: X \rightarrow \ell_{2}^{n}$, and $v: \ell_{2}^{n} \rightarrow Y$, such that $\pi_{2}(u)=1=\|v\|$. Let $C=\sup \left\{\|u a v\|_{1} \mid a \in \mathscr{A}\right\}$. Then $\|T\|_{c b} \geqslant\|u T v\|_{1} /(4 \max \{C, 1\})$.

Proof. Fix $\varepsilon>0$, and find $i \in \mathrm{N}$ s.t. $n=n_{i}$, and $v_{1}\left(u-u_{i}\right)<\varepsilon$, and $4^{-i}<\varepsilon$ (we identify $\ell_{2}^{n}$ with $E_{i}$ ). We view $u$ and $v$ as maps from $X$ to $E_{i}$ and from $E_{i}^{*}$ to $Y$, respectively. By (3.1), $\|v\|_{c b}=\sup \left\{\left\|u_{j} a v\right\|_{c b} \mid j \in \mathrm{N}, a \in \mathscr{A}\right\}$. If $i=j$, then, for any $a \in \mathscr{A}$,

$$
\left\|u_{i} a v\right\|_{c b} \leqslant\left\|u_{i} a v\right\|_{1} \leqslant\|u a v\|_{1}+v_{1}\left(u-u_{i}\right)=C+\varepsilon .
$$

If $j \neq i$

$$
\left\|u_{j} a v\right\|_{c b} \leqslant\left\|u_{j} a v\right\|_{2}=\pi_{2}\left(u_{j} a v\right) \leqslant \pi_{2}\left(u_{j}\right)\|a\|\|v\| \leqslant 1 .
$$


Therefore, $\|v\|_{c b} \leqslant \max \{C+\varepsilon, 1\}$.

By (3.1), $\left\|u_{i}\right\|_{c b}=1$, hence $\|u\|_{c b} \leqslant\left\|u_{i}\right\|_{c b}+v_{1}\left(u-u_{i}\right)<1+\varepsilon$. Therefore,

$$
\|T\|_{c b} \geqslant \frac{\|u T v\|_{c b}}{\|u\|_{c b}\|v\|_{c b}} \geqslant \frac{\|u T v\|_{1}}{(1+\varepsilon)(4+\varepsilon) \max \{C+\varepsilon, 1\}} .
$$

However, $\varepsilon$ can be chosen to be arbitrarily small.

Proof of Theorem 3.1. By (3.1) and the remark following it, $a$ is completely contractive whenever $a \in \mathscr{A}$. By Lemma 3.9, $\|S\|_{c b} \leqslant \pi_{2}(S)$ for any $S \in \Pi_{2}(S)$. This proves part (1) of the theorem.

To prove part (2), pick $T \in C B(X)$ with $\|T\|_{c b}<1 / 4$. Suppose, for the sake of contradiction, that we cannot represent $T=\lambda a+S$, with $a \in \mathscr{A}$, $|\lambda| \leqslant C_{1}$, and $\pi_{2}(S) \leqslant C_{2}$. Then there exist $\gamma>1$, finite dimensional spaces $E_{0}$ and $F_{0}$, and contractions $u_{0} \in B\left(E_{0}, E\right), v \in B\left(E, F_{0}\right)$, s.t. $\pi_{2}\left(v_{0} a u_{0}\right) \leqslant \gamma$ for any $a \in \mathscr{A}$, yet $\pi_{2}\left(v_{0} T u_{0}\right)>\gamma$. By the remark on page 10 of [30], we can assume that $E_{0}=\ell_{2}^{n}$. By trace duality (p. 19 of [30], or [35]), there exists $w \in B\left(F_{0}, \ell_{2}^{n}\right)$, s.t. $\pi_{2}(w)=1$, and $\operatorname{tr}\left(w v_{0} T u_{0}\right)>\gamma$. Moreover, $\left\|w v_{0} a u_{0}\right\|_{1} \leqslant \pi_{2}(w) \pi_{2}\left(v_{0} a u_{0}\right) \leqslant \gamma$ for any $a \in \mathscr{A}$. Lemma 3.10 then yields $\|T\|_{c b} \geqslant 1 / 4$, a contradiction.

\section{Examples and applications}

By Corollary 3.3 (see also Corollary 3.4), an infinite ampliation of any contractive unital weak*-to-weak* continuous representation $\pi: \mathscr{A} \rightarrow B(E)$ gives rise to an operator space $X$, isometric to $\ell_{2}(E)$, s.t. $C B(X)=\pi(\mathscr{A}) \otimes$ $I_{\ell_{2}}+\Pi_{2}(X)$. In some cases, however, we can prove that $\pi(\mathscr{A})$ is $\Pi_{2}$-ASHR without resorting to ampliation. Some examples can be found in [22], [24]. These examples are used to construct "pathological" operator spaces. Below, we present further representations $\pi$ with this property, and operator spaces arising from them (Theorems 4.2, 4.3, 4.6).

\subsection{The long James algebra}

We show that the unit ball of the "long James algebra", viewed as a set of diagonal operators on $\ell_{2}$, is $\Pi_{2}$-ASHR. As a consequence, we construct a separable Hilbertian operator space with a complete transfinite basis, but without the Complete Bounded Approximation Property (and consequently, without a complete basis). This answers the non-commutative version of a question asked in [32]. For the construction, we need the notion of the long James space, introduced in [12]. The construction we present here is slightly different, but it is easy to check that it yields an equivalent norm on the same Banach space. 
Suppose $\eta$ is an infinite ordinal. Denote by $\mathscr{I}_{\eta}$, or simply $\mathscr{I}$, the set of all ordinals $\alpha<\eta$ which are not limit ordinals. For $f: \mathscr{I} \rightarrow \mathrm{C}$, define

$$
\|f\|_{J}=\sup \left\{\left(\sum_{k=1}^{n}\left|f\left(\alpha_{2 k-1}\right)-f\left(\alpha_{2 k}\right)\right|^{2}\right)^{1 / 2} \mid\left(\alpha_{i}\right)_{i=1}^{2 n} \in \mathscr{F}_{n}, n \in \mathrm{N}\right\} .
$$

Here, the set $\mathscr{F}_{n}=\mathscr{F}_{n}(\eta)$ consists of all $2 n$-tuples $\left(\alpha_{i}\right)_{i=1}^{2 n}$ of members of $\mathscr{I}$, for which either $\alpha_{1}<\alpha_{2}<\cdots<\alpha_{2 n-1}<\alpha_{2 n}$, or $\alpha_{2 n}<\alpha_{1}<\alpha_{2}<\cdots<\alpha_{2 n-1}$. Then $\|\cdot\|_{J}$ is a seminorm on $c_{00}(\mathscr{I})+\mathrm{C} \mathbf{1}$, with the kernel $\mathrm{C} \mathbf{1}$. We define by $J(\eta)$ the completion of $c_{00}(\mathscr{I})+\mathrm{C} \mathbf{1}$ with respect to $\|\cdot\|_{J}$.

For the sake of convenience, we introduce a new norm on $J$. Let $\mathscr{I}^{\prime}=$ $\mathscr{I} \cup\{\eta, \eta+\}$. Extend $f: \mathscr{I} \rightarrow \mathrm{C}$ to $\tilde{f}: \mathscr{I}^{\prime} \rightarrow \mathrm{C}$ by setting $\left.\tilde{f}\right|_{\mathscr{I}}=f$, $\tilde{f}(\eta)=\tilde{f}(\eta+)=0$. Set

$\|f\|=\sup \left\{\left(\sum_{k=1}^{n}\left|\tilde{f}\left(\alpha_{2 k-1}\right)-\tilde{f}\left(\alpha_{2 k}\right)\right|^{2}\right)^{1 / 2} \mid\left(\alpha_{i}\right)_{i=1}^{2 n} \in \mathscr{F}_{n}(\eta++), n \in \mathrm{N}\right\}$.

Then $\|\cdot\|$ is a norm on $J(\eta)$. Moreover, any $f \in J(\eta)$ has a unique representation $f_{0}+\lambda \mathbf{1}$, with $\lambda \in \mathrm{C}$ and $f_{0} \in \overline{c_{00}}\|\cdot\|_{J}$. For future reference, we need the following easy inequality: for any $f \in J(\eta)$,

$$
\max \left\{\|f\|_{J},\|f\|_{\infty}\right\} \leqslant\|f\| \leqslant\left(\|f\|_{J}^{2}+2\|f\|_{\infty}^{2}\right)^{1 / 2} .
$$

Next we use the techniques of [2] to make $J(\eta)$ into a commutative unital Banach algebra. For any $f \in \ell_{\infty}(\mathscr{I})$, set $\|f\|_{\mathscr{A}}=\sup \{\|f g\| \mid\|g\| \leqslant 1\}$. We call $\mathscr{A}(\eta)=\left\{f \in \ell_{\infty}(\mathscr{I}) \mid\|f\|_{\mathscr{A}}<\infty\right\}$ the long James algebra. Clearly, it is a unital Banach algebra. The next lemma shows that $\mathscr{A}(\eta)$ and $J(\eta)$ coincide as sets, and describes useful properties of $\mathscr{A}(\eta)$.

Lemma 4.1. Suppose $\eta$ is an infinite ideal.

(1) For any $f \in \ell_{\infty}(\mathscr{I}),\|f\|_{\mathscr{A}} \geqslant\|f\|_{\infty}$.

(2) Suppose $\gamma_{1}<\gamma_{2} \leqslant \eta$ are ordinals. Define

$$
f(\beta)= \begin{cases}1 & \gamma_{1} \leqslant \beta<\gamma_{2}, \\ 0 & \text { otherwise. }\end{cases}
$$

Then either $f=0$, or $\|f\|_{\mathscr{A}}=1$.

(3) $\mathscr{A}(\eta)$ and $J(\eta)$ coincide as sets. For any $f \in J(\eta)$,

$$
\|f\| / \sqrt{2} \leqslant\|f\|_{\mathscr{A}} \leqslant 2\|f\| .
$$


Proof. (1) Fix $\alpha \in \mathscr{I}$, and define $g: \mathscr{I} \rightarrow \mathrm{C}$ by setting

$$
f(\beta)= \begin{cases}1 & \beta=\alpha, \\ 0 & \beta \neq \alpha .\end{cases}
$$

Then $f g=f(\alpha) g$, hence $\|f\|_{\mathscr{A}} \geqslant|f(\alpha)|$.

(2) It suffices to show that, for any $g \in J(\eta)$ and $\left(\alpha_{i}\right)_{i=1}^{2 n} \in \mathscr{F}_{n}(\eta++)$, we have

$$
\sum_{k=1}^{n}\left|\tilde{f}\left(\alpha_{2 k-1}\right) \tilde{g}\left(\alpha_{2 k-1}\right)-\tilde{f}\left(\alpha_{2 k}\right) \tilde{g}\left(\alpha_{2 k}\right)\right|^{2} \leqslant\|g\|^{2} .
$$

We consider the case of $\alpha_{1}<\alpha_{2}<\cdots<\alpha_{2 n}$; the other possibility is handled similarly. By erasing the pairs $\left(\alpha_{2 i-1}, \alpha_{2 i}\right)$ for which $f\left(\alpha_{2 i-1}\right)=f\left(\alpha_{2 i}\right)=0$, we can restrict our attention to one of the following three cases:

(i) $\gamma_{1} \leqslant \alpha_{1}<\alpha_{2}<\cdots<\alpha_{2 n}<\gamma_{2}$;

(ii) $\alpha_{1}<\gamma_{1} \leqslant \alpha_{2}<\cdots<\alpha_{2 n}<\gamma_{2}$;

(iii) $\gamma_{1} \leqslant \alpha_{1}<\alpha_{2}<\cdots<\alpha_{2 n-1}<\gamma_{2} \leqslant \alpha_{2 n}$;

(iv) $\alpha_{1}<\gamma_{1} \leqslant \alpha_{2}<\cdots<\alpha_{2 n-1}<\gamma_{2} \leqslant \alpha_{2 n}$.

In case (i), the inequality (4.2) clearly holds. In case (ii), consider $\alpha_{1}^{\prime}=\eta$, and $\alpha_{i}^{\prime}=\alpha_{i}$ for $2 \leqslant i \leqslant 2 n$. Then $\left(\alpha_{i}^{\prime}\right)_{i=1}^{2 n} \in \mathscr{F}_{n}(\eta++)$, and

$$
\begin{aligned}
\sum_{k=1}^{n}\left|\tilde{f}\left(\alpha_{2 k-1}\right) \tilde{g}\left(\alpha_{2 k-1}\right)-\tilde{f}\left(\alpha_{2 k}\right) \tilde{g}\left(\alpha_{2 k}\right)\right|^{2} & =\sum_{k=1}^{n}\left|\tilde{g}\left(\alpha_{2 k-1}^{\prime}\right)-\tilde{g}\left(\alpha_{2 k}^{\prime}\right)\right|^{2} \\
& \leqslant\|g\|^{2} .
\end{aligned}
$$

The case (iii) is handled in the similar fashion. Finally, if (iv) occurs, we pass to $\alpha_{1}^{\prime}=\eta+, \alpha_{2 n}^{\prime}=\eta$, and $\alpha_{i}^{\prime}=\alpha_{i}$ for $2 \leqslant i \leqslant 2 n-1$.

(3) To show the inequality $\|f\|_{\mathscr{A}} \geqslant\|f\| / \sqrt{2}$, note that, by part (1),

$$
\|f\|_{\mathscr{A}} \geqslant \frac{\|f \mathbf{1}\|}{\|\mathbf{1}\|}=\frac{\|f\|}{\sqrt{2}} .
$$

To obtain the upper estimate on $\|f\|_{\mathscr{A}}$, observe that $\tilde{f} g=\tilde{f} \tilde{g}$. Suppose 
$\left(\alpha_{i}\right)_{i=1}^{2 n} \in \mathscr{F}_{n}(\eta++)$. Then

$$
\begin{aligned}
& \sum_{k=1}^{n}\left|\tilde{f}\left(\alpha_{2 k-1}\right) \tilde{g}\left(\alpha_{2 k-1}\right)-\tilde{f}\left(\alpha_{2 k}\right) \tilde{g}\left(\alpha_{2 k}\right)\right|^{2} \\
& =\sum_{k=1}^{n}\left|\tilde{f}\left(\alpha_{2 k-1}\right)\left(\tilde{g}\left(\alpha_{2 k-1}\right)-\tilde{g}\left(\alpha_{2 k}\right)\right)+\left(\tilde{f}\left(\alpha_{2 k-1}\right)-\tilde{f}\left(\alpha_{2 k}\right)\right) \tilde{g}\left(\alpha_{2 k}\right)\right|^{2} \\
& \leqslant 2 \sum_{k=1}^{n}\left(\left|\tilde{f}\left(\alpha_{2 k-1}\right)\right|^{2}\left|\tilde{g}\left(\alpha_{2 k-1}\right)-\tilde{g}\left(\alpha_{2 k}\right)\right|^{2}+\left|\tilde{f}\left(\alpha_{2 k-1}\right)-\tilde{f}\left(\alpha_{2 k}\right)\right|^{2}\left|\tilde{g}\left(\alpha_{2 k}\right)\right|^{2}\right) \\
& \leqslant 2\left(\|f\|_{\infty}^{2}\|g\|^{2}+\|f\|^{2}\|g\|_{\infty}^{2}\right) \leqslant 4\|f\|^{2}\|g\|^{2}
\end{aligned}
$$

(here, we use the left hand side of (4.1). Taking the supremum over all $\left(\alpha_{i}\right)_{i=1}^{2 n} \in$ $\mathscr{F}_{n}(\eta++)$, we obtain the inequality $\|f g\| \leqslant 2\|f\|\|g\|$, as desired.

Denote by $\left(e_{\alpha}\right)_{\alpha \in \mathscr{I}}$ the canonical orthonormal basis in $\ell_{2}(\mathscr{I})$. Consider the "natural" representation $\pi: \mathscr{A}(\eta) \rightarrow B\left(\ell_{2}(\eta)\right)$ by setting $\pi(f) e_{\alpha}=$ $f(\alpha) e_{\alpha}$. It follows from the discussion above that $\pi$ is a unital contractive representation. A particular case of the following lemma is implicit in [22].

Theorem 4.2. Suppose $\eta$ is an infinite ordinal. Then $\pi(\mathrm{Ba}(\mathscr{A}(\eta)))$ is $(10 \sqrt{6}, 4)-\Pi_{2}$-ASHR.

Proof. For the sake of brevity, let $H=\ell_{2}(\mathscr{I})$. Suppose $T \in B(H)$ is such that $\|u T v\|_{2}<1 / 5$ whenever the finite rank contractions $u$ and $v$ satisfy $\|u \pi(a) v\|_{2} \leqslant 1$ for any $a \in \operatorname{Ba}(\mathscr{A}(\eta))$. We shall show that $T=T_{1}+\pi(f)$, where $\left\|T_{1}\right\|_{2} \leqslant 4 / 5$, and $\|f\|_{\mathscr{A}} \leqslant 2 \sqrt{6}$.

For $\alpha \in \mathscr{I}$, set $f(\alpha)=\left\langle T e_{\alpha}, e_{\alpha}\right\rangle$. Let $T_{0}$ be the diagonal part of $T$ (that is, $T_{0} e_{\alpha}=f(\alpha) e_{\alpha}$ for any $\alpha \in \mathscr{I}$ ), and let $T_{1}=T-T_{0}$ be the off-diagonal part. Suppose $S_{1}$ and $S_{2}$ are disjoint finite subsets of $\mathscr{I}$. Let $P_{S_{1}}$ and $P_{S_{2}}$ be the corresponding orthogonal projections. Then $P_{S_{1}} \pi(a) P_{S_{2}}=0$ for any $a \in \mathrm{Ba}(\mathscr{A}(\eta))$, hence $\left\|P_{S_{1}} T P_{S_{2}}\right\|_{2}<1 / 5$. An averaging (cf. the proof of Lemma 2.3) argument yields $\left\|T_{1}\right\|_{2} \leqslant 4 / 5$. Moreover, if the finite rank contractions $u$ and $v$ satisfy $\|u \pi(a) v\|_{2} \leqslant 1$ for any $a \in \operatorname{Ba}(\mathscr{A}(\eta))$, then $\left\|u T_{0} v\right\|_{2} \leqslant\|u T v\|_{2}+\left\|T_{1}\right\|_{2} \leqslant 1$.

Recall that $T_{0} e_{\alpha}=f(\alpha) e_{\alpha}$. It suffices to show that $\|f\|_{\mathscr{A}} \leqslant 2 \sqrt{6}$. To this end, note first that $\|f\|_{\infty} \leqslant 1$. Indeed, otherwise there exists $\alpha \in \mathscr{I}$ s.t. $|f(\alpha)|>1$. Let $u$ and $v$ be equal to the orthogonal projection onto $C_{\alpha}$. Then $\|u \pi(a) v\|_{2} \leqslant 1$ for any $a \in \operatorname{Ba}(\mathscr{A}(\eta))$, yet $\left\|u T_{0} v\right\|_{2}>1$, a contradiction.

Next consider $\left(\alpha_{i}\right)_{i=1}^{2 n} \in \mathscr{F}_{n}$, and show that $\sum_{k=1}^{n}\left|f\left(\alpha_{2 k-1}\right)-f\left(\alpha_{2 k}\right)\right|^{2} \leqslant 4$ (and therefore, $\|f\|_{J} \leqslant 2$ ). Indeed, denote by $\left(\delta_{k}\right)_{k=1}^{n}$ an orthonormal basis in $\ell_{2}^{n}$, and consider

$$
v: \ell_{2}^{n} \rightarrow H: \delta_{k} \mapsto\left(e_{\alpha_{2 k-1}}+e_{\alpha_{2 k}}\right) / \sqrt{2},
$$


and

$$
u: H \rightarrow \ell_{2}^{n}: \xi \mapsto \sum_{k=1}^{n} \frac{1}{\sqrt{2}}\left\langle\xi, e_{\alpha_{2 k-1}}-e_{\alpha_{2 k}}\right\rangle \delta_{k} .
$$

If $S$ is a diagonal operator with $S e_{\alpha}=g(\alpha) e_{\alpha}$ for $\alpha \in \mathscr{I}$, then

$$
u S v \delta_{k}=\frac{1}{2}\left(g\left(\alpha_{2 k-1}\right)-g\left(\alpha_{2 k}\right)\right) \delta_{k},
$$

hence

$$
\|u S v\|_{2}^{2}=\sum_{k=1}^{n}\left|g\left(\alpha_{2 k-1}\right)-g\left(\alpha_{2 k}\right)\right|^{2} / 4 .
$$

Then, for any $a \in \mathscr{A}(\eta),\|u \pi(a) v\|_{2} \leqslant\|a\|_{J} \leqslant\|a\|<1$, by (4.1) and Lemma 4.1. Thus, $\left\|u T_{0} v\right\|_{2} \leqslant 1$. By (4.3), $\|f\|_{J} \leqslant 2$. By (4.1), $\|f\| \leqslant \sqrt{6}$. By Lemma 4.1, $\|f\|_{\mathscr{A}} \leqslant 2 \sqrt{6}$.

\subsection{Transfinite bases}

The notion of a transfinite basis in a Banach space was introduced by C. Bessaga [4]. We refer the reader to that paper, and to Section 2 of [32], for more information. Here we present a non-commutative version of the same definition. Suppose $X$ is an operator space, $\eta$ is an ordinal, and $\left(P_{\gamma}\right)_{0 \leqslant \gamma \leqslant \eta}$ is a family of projections on $X$ such that $\sup _{\gamma}\left\|P_{\gamma}\right\|_{c b}<\infty, P_{\alpha} P_{\beta}=P_{\min \{\alpha, \beta\}}, P_{0}=0$, $P_{\eta}=I_{X}$, and for each $x \in X$, the map $\gamma \mapsto P_{\gamma} x$ is continuous on $[0, \eta]$ (here, $[0, \eta]$ and $X$ are equipped with the order, resp. norm, topology). Then the family $\left(P_{\gamma}\right)_{0 \leqslant \gamma \leqslant \eta}$ is called a complete transfinite Schauder decomposition (of length $\eta)$. If, in addition, $\operatorname{dim}\left(P_{\gamma+}-P_{\gamma}\right)=1$ for any $\gamma$, then $\left(P_{\gamma}\right)_{0 \leqslant \gamma \leqslant \eta}$ is called a complete transfinite (Schauder) basis (of length $\eta$ ). Note that (complete) transfinite bases of length $\omega$ (the first infinite ordinal) in Banach (operator) spaces are just the usual (complete) bases.

Recall that an operator space $X$ is said to have the Completely Bounded Compact Approximation Property (CBCAP for short) if there exists a constant $C$ s.t. for every finite dimensional $E \hookrightarrow X$ there exists a compact operator $T \in C B(X)$ s.t. $\|T\|_{c b} \leqslant C$, and $\left.T\right|_{E}=I_{E}$. The definition of the Completely Bounded Approximation Property (CBAP) is similar, but more restrictive: the operators $T$ are required to have finite rank. For Banach spaces, the Bounded (Compact) Approximation Property (BAP, CBAP) are defined in a similar fashion.

Clearly, every operator (Banach) space with a (complete) basis has the CBAP (resp. BAP). It was shown in [32] that every Banach space with a transfinite basis has the Approximation Property (which is strictly weaker 
that the BAP - see e.g. [5] for the connections between the two properties). The problem of whether the existence of the transfinite basis implies the Bounded Approximation Property is open. We give a negative answer to the non-commutative version of this question.

THEOREM 4.3. There exists an operator space with a transfinite basis, failing the Completely Bounded Compact Approximation Property.

Proof. Consider the ordinal $\eta=\omega^{2}$. For simplicity, let $\mathscr{A}=\mathscr{A}(\eta)$. In the previous subsection, it is shown that $\pi(\mathrm{Ba}(\mathscr{A}))$ is $\Pi_{2}$-ASHR. We build an operator space $X$ "around" this set. More precisely, we use the results of Chapter 3 to construct an operator space $X$, isometric to $\ell_{2}(\mathscr{I})$ on the Banach space level, such that $T \in B(X)$ is completely bounded iff $T \in \pi(\mathscr{A})+\mathscr{S}_{2}$. Moreover, for any $a \in \mathscr{A}$ and $S \in \mathscr{T}_{2}$, we have $\|\pi(a)+S\|_{c b} \leqslant\|a\|_{\mathscr{A}}+\|S\|_{2}$, while there exists a constant $c$ s.t. every $T \in C B(X)$ can be represented as $T=\pi(a)+S$, with $\|a\|_{\mathscr{A}}+\|S\|_{2} \geqslant c\|T\|_{c b}$.

For $\gamma \leqslant \eta$, define $f_{\gamma}$ by setting, for $\alpha \in \mathscr{I}$,

$$
f_{\gamma}(\alpha)= \begin{cases}1 & \alpha<\gamma \\ 0 & \alpha \geqslant \gamma\end{cases}
$$

By Lemma 4.1(b), $\left\|f_{\gamma}\right\|_{\mathscr{A}}=1$ unless $\gamma=1$, and $\left\|\mathbf{1}-f_{\gamma}\right\|_{\mathscr{A}}=1$ unless $\gamma=\eta$.

Therefore, for any $\gamma \leqslant \eta$, the projection $P_{\gamma}=\pi\left(f_{\gamma}\right)$ is completely contractive. Moreover,

$$
P_{\gamma} e_{\alpha}= \begin{cases}e_{\alpha} & \alpha<\gamma \\ 0 & \alpha \geqslant \gamma\end{cases}
$$

for any $\alpha \in \mathscr{I}$. Thus, the family $\left(P_{\gamma}\right)$ defines a completely transfinite basis on $X$.

Suppose, for the sake of contradiction, that $X$ has the CBCAP. For each $n$, consider $E_{n}=\operatorname{span}\left[e_{\omega}, e_{2 \omega}, \ldots, e_{n \omega}\right] \hookrightarrow X$. Then there exists a compact operator $T=\pi(f)+S$ s.t. $\|f\|_{\mathscr{A}}+\|S\|_{2}<C$ (C is a constant $)$, and $\left.T\right|_{E_{n}}=$ $I_{E_{n}}$. In particular,

$$
\sum_{j=1}^{n}|f(j \omega)-1|^{2}=\sum_{j=1}^{n}\left\|S e_{j}\right\|^{2} \leqslant\|S\|_{2}^{2}<C^{2} .
$$

Suppose $n>32 C^{2}$. Then the set

$$
\mathscr{G}_{n}=\{1 \leqslant j \leqslant n|| f(j \omega)-1 \mid<1 / 4\}
$$

is such that $\left|\mathscr{G}_{n}^{c}\right|<16 C^{2}$. 
On the other hand, $\pi(f)$ is compact, hence for any $j \in[1, n]$, there exists $\alpha_{j} \in(j \omega,(j+1) \omega)$ s.t. $\left|f\left(\alpha_{j}\right)\right|<1 / 4$. Then

$$
\|f\|^{2} \geqslant \sum_{j \in \mathscr{G}_{n}}\left|f\left(\alpha_{j}\right)-f(j \omega)\right|^{2} \geqslant \frac{\left|\mathscr{G}_{n}\right|}{4} \geqslant \frac{n}{8} .
$$

By Lemma 4.1, $\|f\|_{\mathscr{A}} \geqslant \sqrt{n} / 4$, which yields the desired contradiction.

Remark 4.4. As in Section 2 of [32], we can show that any operator space with a complete transfinite basis has the Strong Operator Approximation Property (SOAP). Thus, Theorem 4.3 yields an example of an operator space with the SOAP, but without the CBCAP. The classical counterpart of this result is the existence of Banach spaces with the Approximation Property, but without the Bounded Compact Approximation Properties (see Section 8 of [5] for the examples).

REMARK 4.5. We can generalize the definition of the long James space in the following way. Suppose $\mathscr{E}$ is a symmetric separable sequence space, which is mononormalizing (in the terminology of p. 88 of [14]; see also Section 3 of [23]). That is, we require that two conditions be satisfied: (i) if $\lim _{n}\left\|\left(x_{1}, \ldots, x_{n}, 0,0, \ldots\right)\right\|_{\mathscr{E}}=C<\infty$, then $\left(x_{i}\right)_{i \in \mathrm{N}} \in \mathscr{E}$, and $\left\|\left(x_{i}\right)_{i \in \mathrm{N}}\right\|_{\mathscr{E}}=$ $C$; and (ii) if $\left(x_{i}\right)_{i \in \mathrm{N}} \in \mathscr{E}$, then $\lim _{n}\left\|\left(x_{n}, x_{n+1}, \ldots\right)\right\|_{\mathscr{E}}=0$. The space $\mathscr{E}$ is nice if, in addition, $\lim _{n}\|(1, \ldots, 1,0, \ldots)\|_{\mathscr{E}}=\infty$.

We denote by $\mathscr{S}_{\mathscr{E}}$ the operator ideal, corresponding to a nice space $\mathscr{E}$ : for a compact operator $T$, denote by $\left(\sigma_{i}(T)\right)$ the sequence of singular numbers of $T$, and set $\|T\|_{\mathscr{E}}=\left\|\left(\sigma_{i}(T)\right)\right\|_{\mathscr{E}}$.

For any infinite ordinal $\eta$, and for any nice sequence space $\mathscr{E}$, we define a modified long James space: for $f: \mathscr{I} \rightarrow \mathrm{C}$, define

$$
\|f\|_{J_{\mathscr{E}}}=\sup \left\{\left\|\left(f\left(\alpha_{2 k-1}\right)-f\left(\alpha_{2 k}\right)_{1 \leqslant k \leqslant n}\right)\right\|_{\mathscr{E}} \mid\left(\alpha_{i}\right)_{i=1}^{2 n} \in \mathscr{F}_{n}, n \in \mathrm{N}\right\} .
$$

Here, the set $\mathscr{F}_{n}=\mathscr{F}_{n}(\eta)$ consists of all $2 n$-tuples $\left(\alpha_{i}\right)_{i=1}^{2 n}$ of members of $\mathscr{I}$, for which either $\alpha_{1}<\alpha_{2}<\cdots<\alpha_{2 n-1}<\alpha_{2 n}$, or $\alpha_{2 n}<\alpha_{1}<\alpha_{2}<\cdots<\alpha_{2 n-1}$. Then $\|\cdot\|_{J_{\mathscr{\delta}}}$ is a seminorm on $c_{00}(\mathscr{I})+\mathbf{C} \mathbf{1}$, with the kernel $\mathbf{C} \mathbf{1}$. We define by $J_{\mathscr{E}}(\eta)$ the completion of $c_{00}(\mathscr{I})+\mathrm{C} \mathbf{1}$ with respect to $\|\cdot\|_{J_{\mathscr{E}}}$.

Similarly, we define on $c_{00}+\mathrm{C} \mathbf{1}$ the norms $\|\cdot\|_{\mathscr{E}}$ and $\|\cdot\|_{\mathscr{A} \mathscr{E}(\eta)}$ (analogues of $\|\cdot\|$ and $\left.\|\cdot\|_{\mathscr{A}(\eta)}\right)$. As above, we show that the norms $\|\cdot\|_{\mathscr{E}}$ and $\|\cdot\|_{\mathscr{A}_{\mathscr{E}}(\eta)}$ are equivalent to each other, and both of them are equivalent to $\|\cdot\|_{J_{\delta}}$ on $c_{00}$.

Denote by $\pi_{\mathscr{E}}$ the identity representation of $\mathscr{A}_{\mathscr{E}}(\eta)$ on the diagonal of $B\left(\ell_{2}(\mathscr{I})\right)$. As before, we show that $\pi_{\mathscr{E}}\left(\mathrm{Ba}\left(\mathscr{A}_{\mathscr{E}}(\eta)\right)\right)$ is $\mathscr{S}_{\mathscr{E}}$-ASHR.

Now suppose the ordinal $\eta$ is countable, and the formal identity map $\ell_{2} \rightarrow \mathscr{E}$ is bounded. By [23], we can equip $\ell_{2}(\mathscr{I})$ with an operator space structure $X$, s.t. $C B(X)=\pi\left(\mathscr{A}_{\mathscr{E}}(\eta)\right)+\mathscr{S}_{\mathscr{E}}\left(\ell_{2}(\mathscr{I})\right)$. 


\subsection{Almost multiplicative functionals}

An old problem in Banach algebras asks whether every "almost multiplicative" linear functional on a given Banach algebra is near multiplicative. More precisely: suppose $\mathscr{A}$ is a Banach algebra. We say that $f \in \mathscr{A}^{*}$ is $\delta$-multiplicative $|f(a) f(b)-f(a b)|<\delta\|a\|\|b\|$ for any $a, b \in \mathscr{A}$. 0-multiplicative functionals are referred to simply as multiplicative. A Banach algebra $\mathscr{A}$ is called AMNM (almost multiplicative near multiplicative) if there exists a function $\varepsilon$ on $(0, \infty)$, with $\lim _{\delta \rightarrow 0} \varepsilon(\delta)=0$, such that for any $\delta$-multiplicative $f \in \mathscr{A}^{*}$ there exists a multiplicative $g \in \mathscr{A}^{*}$ s.t. $\|f-g\|<\varepsilon(\delta)$.

Describing the AMNM Banach algebras is a long-standing open problem. There reader is referred to [16], [17], and references therein, for a brief introduction. Here, we mention several results: (1) The algebras $C_{0}(K)$ ( $K$ locally compact Hausdorff) are AMNM. (2) The disk algebra is AMNM. Moreover, any singly generated uniform algebra is AMNM [15] (3) There exists a uniform algebra which is not AMNM [34]. (4) If $G$ is a locally compact abelian group, then $L_{1}(G)$ is AMNM. (5) It is not known whether $H^{\infty}$ is AMNM (that is, whether $H^{\infty}$ has "almost corona").

No examples of Banach spaces $E$ for which $B(E)$ is not AMNM are known. Note that, if $E$ is isomorphic to $\ell_{\infty}^{n}(E)$ for some $n$, then $B(E)$ is AMNM. Indeed, in this case, there exist idempotents $p_{1}, \ldots, p_{n} \in B(E)$, s.t. $p_{i} p_{j}=$ $\delta_{i j} p_{i}$, and $\sum_{i=1}^{n} p_{i}=I_{E}$, and $u_{1}, v_{1}, \ldots, u_{n}, v_{n} \in B(E)$ s.t. $u_{i} v_{i}=p_{i}$, and $v_{i} u_{i}=I_{E}$. Let $K=\max _{i}\left\|u_{i}\right\|\left\|v_{i}\right\|$. If $f \in B(E)^{*}$ is $\delta$-multiplicative, then $\left|f\left(I_{E}\right)^{2}-f\left(I_{E}\right)\right| \leqslant \delta$. Therefore, either $\left|f\left(I_{E}\right)\right|<\phi(\delta)$, or $\left|f\left(I_{E}\right)-1\right|<$ $\phi(\delta)$, where $\phi$ is a continuous map on $[0, \infty)$, s.t. $\phi(0)=0$. In the former case,

$$
\left|f\left(T I_{E}\right)-f(T) f\left(I_{E}\right)\right|=|f(T)|\left|1-f\left(I_{E}\right)\right| \leqslant \phi(\delta)\|T\|
$$

for any $T \in B(E)$, hence $\|f\| \leqslant \phi(\delta) /(1-\phi(\delta))$. The latter case can be ruled out as follows: if $\left|f\left(I_{E}\right)-1\right|<\phi(\delta)$, then

$$
\left|f\left(u_{i}\right) f\left(v_{i}\right)-1\right| \leqslant\left|f\left(v_{i}\right) f\left(u_{i}\right)-f\left(I_{E}\right)\right|+\left|f\left(I_{E}\right)-1\right| \leqslant K \delta+\phi(\delta) .
$$

However, $\left|f\left(u_{i}\right) f\left(v_{i}\right)-f\left(p_{i}\right)\right| \leqslant K \delta$, hence $\left|f\left(p_{i}\right)-1\right| \leqslant 2 K \delta+\phi(\delta)$ for each $i$. Then

$$
n-1-\phi(\delta) \leqslant\left|f\left(I_{E}\right)-n\right| \leqslant \sum_{i}\left|f\left(p_{i}\right)-1\right| \leqslant n(2 K \delta+\phi(\delta))
$$

which is clearly impossible for $\delta$ small enough.

The spaces $E$ which are isomorphic to $\ell_{\infty}^{n}(E)$ include the Orlitz spaces, the Schatten spaces, and more. 
For many classes of Banach spaces, however, it is not known whether $B(E)$ is AMNM. For instance, suppose $E$ a hereditarily indecomposable space. Then for every $T \in B(E)$ there exists a (unique) $\lambda_{T} \in \mathrm{C}$ s.t. $T-\lambda_{T} I_{E}$ is strictly singular. It is well-known (see e.g. [18]) that the spectrum of any strictly singular operator is countable, 0 its only possible cluster point, and all isolated points are finite rank eigenvalues. By [1], $f(S)=0$ whenever $f \in B(E)^{*}$ is multiplicative, and $S$ is finite rank. Moreover, by [1] again, $f(S) \in \sigma(S)$ whenever $S \in B(E)$, and $f$ is multiplicative.

This shows that the only non-trivial multiplicative functional on $B(E)$, with $E$ as above, is defined by $f(T)=\lambda_{T}$, with $\lambda_{T}$ defined in the previous paragraph. We have no good description of $\delta$-multiplicative members of $B(E)^{*}$.

The same reasoning could be repeated for the algebras $C B(X)$. In this case, however, we can exhibit an example of $X$ s.t. $C B(X)$ is not AMNM.

Theorem 4.6. There exists an operator space $X$ such that $C B(X)$ is not AMNM.

Proof. We recapitulate B. Johnson's construction (Example 9.1 of [16]). There, it is shown that there exists a sequence of unital Banach algebras $\mathscr{A}_{n}$ s.t., for each $n \in \mathrm{N}$, (1) $\mathscr{A}_{n}$ is $\mathrm{C}^{n}$ with pointwise multiplication; (2) there exists $f_{n} \in \mathscr{A}_{n}^{*}$ s.t. $\left\|f_{n}\right\|=f\left(\mathbf{1}_{n}\right)\left(\mathbf{1}_{n}\right.$ is the identity of the algebra $\left.\mathscr{A}_{n}\right)$; (3) $\left|f_{n}(a) f_{n}(b)-f_{n}(a b)\right| \leqslant\|a\|\|b\| / n$ for any $a, b \in \mathscr{A}_{n} ;(4)\left\|f_{n}-f\right\| \geqslant 1-1 / n$ whenever $f \in \mathscr{A}_{n}^{*}$ is multiplicative.

Consider now the Banach algebra $\mathscr{A}=\mathrm{C} \mathbf{1} \oplus_{1}\left(\sum_{i} \mathscr{A}_{i}\right)_{\ell_{1}}$. It has a predual $A_{*}=\mathrm{C} \oplus_{\infty}\left(\sum_{i} \mathscr{A}_{i}^{*}\right)_{c_{0}}$. We shall show that the multiplication in $\mathscr{A}$ is weak ${ }^{*}$ continuous. By commutativity, it suffices to show the following: suppose $y \in$ $\mathscr{A}$, and a net $\left(x_{\alpha}\right)$ converges weak* to $x \in \mathscr{A}$. Then $x_{\alpha} y \rightarrow x y$ weak* By triviality of multiplication by $\mathbf{1}$, we it suffices to consider $y=\left(y_{i}\right)$, with $y_{i} \in \mathscr{A}_{i}$. Furthermore, write $x=a \mathbf{1}+\left(x_{i}\right)$, and $x_{\alpha}=a_{\alpha} \mathbf{1}+\left(x_{\alpha i}\right)$. Clearly, $\lim _{\alpha} a_{\alpha}=a$. Therefore, we can assume $a=0$, and $a_{\alpha}=0$ for each $\alpha$. Then $x y=\left(x_{i} y_{i}\right)$, and $x_{\alpha} y=\left(x_{\alpha i} y_{i}\right)$. We have to show that, for any $\left(z_{i}\right) \in$ $\left(\sum \mathscr{A}_{i}^{*}\right)_{c_{0}}$

$$
\lim _{\alpha} \sum_{i}\left\langle x_{\alpha i} y_{i}, z_{i}\right\rangle=\sum_{i}\left\langle x_{i} y_{i}, z_{i}\right\rangle .
$$

We can identify $\mathscr{A}_{i}^{*}$ with $C^{n}$ as a vector space. Let $z_{i}^{\prime}=y_{i} z_{i}$ (with pointwise multiplication). A standard Banach algebra calculation yields $\left\|z_{i}^{\prime}\right\| \leqslant$ $\left\|y_{i}\right\|\left\|z_{i}\right\|$. Therefore, $z^{\prime}=\left(z_{i}^{\prime}\right) \in\left(\sum \mathscr{A}_{i}^{*}\right)_{c_{0}}$. Thus,

$$
\lim _{\alpha} \sum_{i}\left\langle x_{\alpha i} y_{i}, z_{i}\right\rangle=\lim _{\alpha}\left\langle x_{\alpha}, z^{\prime}\right\rangle=\left\langle x z^{\prime}\right\rangle=\sum_{i}\left\langle x_{i} y_{i}, z_{i}\right\rangle,
$$

as desired. 
Now extend the functional $f_{n} \in \mathscr{A}_{n}^{*}$ to the whole algebra $\mathscr{A}$, by setting $g_{n}\left(a \mathbf{1} \oplus\left(x_{i}\right)_{i \in \mathrm{N}}\right)=a+f_{n}\left(x_{n}\right)$ (here, $\left.x_{i} \in \mathscr{A}_{i}\right)$. By definition, $\left\|g_{n}\right\|=1$. We shall show that $g_{n}$ is $n^{-1}$-multiplicative. Indeed, consider norm $1 x=a \mathbf{1} \oplus\left(x_{i}\right)$ and $y=b \mathbf{1} \oplus\left(y_{i}\right)$. Then $g_{n}(x)=a+f_{n}\left(x_{n}\right), g_{n}(y)=b_{n}+f_{n}\left(y_{n}\right), g_{n}(x y)=$ $a b+a f\left(y_{n}\right)+b f\left(x_{n}\right)+f_{n}\left(x_{n} y_{n}\right)$, and

$$
\left|g_{n}(x) g_{n}(y)-g_{n}(x y)\right|=\left|f_{n}\left(x_{n}\right) f_{n}\left(y_{n}\right)-f_{n}\left(x_{n} y_{n}\right)\right| \leqslant n^{-1}\left\|x_{n}\right\|\left\|y_{n}\right\| \leqslant n^{-1} \text {. }
$$

The last inequality shows that $g_{n}$ is $n^{-1}$-multiplicative.

Next we show that $\left\|g_{n}-g\right\| \geqslant 1-n^{-1}$ whenever $g \in \mathscr{A}^{*}$ is multiplicative. Indeed, then $\left.g\right|_{\mathscr{A}_{n}}$ is also multiplicative, and $\left\|g_{n}-g\right\| \geqslant\left\|f_{n}-\left.g\right|_{\mathscr{A}_{n}}\right\| \geqslant 1-n^{-1}$, per the result of B. Johnson described above.

By Corollary 3.4, there exists a Banach space $E$, an operator space $X$, isometric to $\ell_{2}(E)$, and a unital isometric representation $\pi: \mathscr{A} \rightarrow B(E)$ s.t. $C B(X)=\pi(\mathscr{A}) \otimes I_{\ell_{2}}+\Pi_{2}(X)$. For $n \in \mathrm{N}$, define $\tilde{g}_{n} \in C B(X)^{*}$ by setting $\tilde{g}_{n}\left(\pi(a) \otimes I_{\ell_{2}}+S\right)=g_{n}(a)$ whenever $a \in \mathscr{A}$, and $S$ is 2-summing. Clearly, $\tilde{g}_{n}$ is well-defined, $\left\|\tilde{g}_{n}\right\|=1$, and $\tilde{g}_{n}$ is $n^{-1}$-multiplicative. Moreover, for any multiplicative $\tilde{g} \in C B(X)^{*}$, define $g \in \mathscr{A}^{*}$ by setting $g(a)=\tilde{g}\left(\pi(a) \otimes I_{\ell_{2}}\right)$. Then $\left\|\tilde{g}_{n}-\tilde{g}\right\| \geqslant\left\|g_{n}-g\right\| \geqslant 1-n^{-1}$, which shows that $C B(X)$ is not AMNM.

ACKNOWLEDGEMENT. Part of this work was carried out while the author was visiting the University of Illinois at Urbana-Champaign. The author wishes to thank UIUC for its hospitality. The author is grateful to the referee for contributing useful suggestions, and for bringing [9] to his attention.

\section{REFERENCES}

1. Abramovich, Yu. A., and Aliprantis, C., An Invitation to Operator Theory, Graduate Studies in Math. 50, Amer. Math. Soc., Providence, RI 2002.

2. Andrew, A., and Green, W., On James' quasi-reflexive Banach space as a Banach algebra, Canad. J. Math. 32 (1980), 1080-1101.

3. Berkson, E., and Porta, H., Representations of $\mathfrak{B}(X)$, J. Funct. Anal. 3 (1969), 1-34.

4. Bessaga, C., Topological equivalence of unseparable reflexive Banach spaces. Ordinal resolutions of identity and monotone bases, Bull. Acad. Polon. Sci. Sér. Sci. Math. Astronom. Phys. 15 (1967), 397-399.

5. Casazza, P., Approximation properties, pp. 271-316 in: Handbook of the Geometry of Banach Spaces, Vol. 1, North-Holland, Amsterdam 2001.

6. K. Davidson, Nest Algebras. Triangular Forms for Operator Algebras on Hilbert Space, Pitman Research Notes Math. 191, Longman, Harlow 1988.

7. Davidson, K., and Szarek, S., Local operator theory, random matrices and Banach spaces, 317-366 in: Handbook of the Geometry of Banach Spaces, Vol. 1, North-Holland, Amsterdam 2001.

8. Daws, M., Dual Banach algebras: representations and injectivity, Studia Math. 178 (2007), 231-275.

9. Daws, M., Weakly almost periodic functionals, representations, and operator spaces, preprint. 
10. Defant, A., and Floret, K., Tensor Norms and Operator Ideals, North-Holland Math. Studies 176, North-Holland, Amsterdam 1993.

11. Diestel, J., Jarchow, H., and Tonge, A., Absolutely Summing Operators, Cambridge Studies in Adv. Math. 43, Cambridge University Press, Cambridge 1995.

12. Edgar, G., A long James space, pp. 31-37 in: Measure Theory, Oberwolfach 1979, Lecture Notes in Math. 794, Springer, Berlin 1980.

13. Effros, E., and Ruan, Z.-J., Operator Spaces, London Math. Soc. Math. Monographs (N.S.) 23, Oxford University Press, New York 2000.

14. Gohberg, I. C., and Kreĭn, M. G., Introduction to the Theory of Linear Nonselfadjoint Operators, Translations of Math. Monographs 18, Amer. Math. Soc., Providence, RI 1969.

15. Jarosz, K., Almost multiplicative functionals, Studia Math. 124 (1997), 37-58.

16. Johnson, B., Approximately multiplicative functionals, J. London Math. Soc. (2) 34 (1986), 489-510.

17. Johnson, B., Approximately multiplicative maps between Banach algebras, J. London Math. Soc. (2) 37 (1988), 294-316.

18. Lindenstrauss, J., and Tzafriri, L., Classical Banach Spaces I. Sequence Spaces, Ergebnisse der Math. 92, Springer, Berlin 1977.

19. Müller, V., and Ptak, M., Hyperreflexivity offinite-dimensional subspaces, J. Funct. Anal. 218 (2005), 395-408.

20. Neufang, M., Ruan, Z.-J., and Spronk, N., Completely isometric representations of $M_{\mathrm{cb}} A(G)$ and $\operatorname{UCB}(\hat{G})^{*}$, Trans. Amer. Math. Soc. 360 (2008), 1133-1161.

21. Oikhberg, T., Operator spaces with prescribed sets of completely bounded maps, J. Funct. Anal. 224 (2005), 296-315.

22. Oikhberg,T., Operator spaces with complete bases, lacking completely unconditional bases, Houston J. Math. 32 (2006), 551-561.

23. Oikhberg, T., Hyperreflexivity and operator ideals, J. Funct. Anal. 246 (2007), 242-280.

24. Oikhberg, T., The operator shift space, Proc. Edinburgh Math. Soc. (2) 51 (2008), 229-263.

25. Oikhberg, T., and Ricard, E., Operator spaces with few completely bounded maps, Math. Ann. 328 (2004), 229-259.

26. Ovsepian, R., and Pełczyński, A., On the existence of a fundamental total and bounded biorthogonal sequence in every separable Banach space, and related constructions of uniformly bounded orthonormal systems in $L^{2}$, Studia Math. 54 (1975), 149-159.

27. Paulsen, V., Completely Bounded Maps and Operator Algebras, Cambridge Studies in Adv. Math. 78, Cambridge University Press, Cambridge 2002.

28. Pełczyński, A., All separable Banach spaces admit for every $\varepsilon>0$ fundamental total and bounded by $1+\varepsilon$ biorthogonal sequences, Studia Math. 55 (1976), 295-304.

29. Pietsch, A., Operator Ideals, North-Holland Math. Library 20, North-Holland, Amsterdam 1980.

30. Pisier, G., Factorization of Linear Operators and Geometry of Banach Spaces, CBMS Regional Conference Series in Math. 60, Amer. Math. Soc., Providence, RI 1986.

31. Pisier, G., Introduction to Operator Space Theory, London Math. Soc. Lecture Notes 294, Cambridge University Press, Cambridge 2003.

32. Rosenthal, H., Contractively complemented subspaces of Banach spaces with reverse monotone (transfinite) bases, pp. 1-14 in: Texas Functional Analysis Seminar 1984-1985 (Austin, TX), Longhorn Notes, Univ. Texas Press, Austin, TX 1985.

33. Runde, V., Lectures on Amenability, Lecture Notes in Math. 1774, Springer, Berlin 2002.

34. Sidney, S., Are all uniform algebras AMNM?, Bull. London Math. Soc. 29 (1997), 327-330.

35. Tomczak-Jaegermann, N., Banach-Mazur Distances and Finite-Dimensional Operator Ideals, Pitman Monographs Pure Appl. Math. 38, Longman, Harlow 1989. 
36. Uygul, F., A representation theorem for completely contractive dual Banach algebras, preprint.

DEPARTMENT OF MATHEMATICS

UNIVERSITY OF CALIFORNIA - IRVINE

IRVINE CA 92697

U. S. A.

E-mail: toikhber@math.uci.edu 